\title{
Nucleus accumbens GABAergic inhibition generates intense eating and fear that resists environmental retuning and needs no local dopamine
}

\author{
Jocelyn M. Richard, ${ }^{1, \star}$ Andrea M. Plawecki ${ }^{1}$ and Kent C. Berridge ${ }^{1}$ \\ ${ }^{1}$ Department of Psychology, University of Michigan, Ann Arbor, MI, USA
}

Keywords: accumbens shell, eating, fear, GABA, glutamate, rat

\begin{abstract}
Intense fearful behavior and/or intense appetitive eating behavior can be generated by localized amino acid inhibitions along a rostrocaudal anatomical gradient within medial shell of nucleus accumbens of the rat. This can be produced by microinjections in medial shell of either the $\gamma$-aminobutyric acid $(\mathrm{GABA})_{\mathrm{A}}$ agonist muscimol (mimicking intrinsic GABAergic inputs) or the AMPA $(\alpha-$ amino-3-hydroxy-5-methylisoxazole-4-propionic acid) antagonist DNQX (6,7-dinitroquinoxaline-2,3-dione), disrupting corticolimbic glutamate inputs). At rostral sites in medial shell, each drug robustly stimulates appetitive eating and food intake, whereas at more caudal sites the same drugs instead produce increasingly fearful behaviors such as escape, distress vocalizations and defensive treading (an antipredator behavior rodents emit to snakes and scorpions). Previously we showed that intense motivated behaviors generated by glutamate blockade require local endogenous dopamine and can be modulated in valence by environmental ambience. Here we investigated whether GABAergic generation of intense appetitive and fearful motivations similarly depends on local dopamine signals, and whether the valence of motivations generated by GABAergic inhibition can also be retuned by changes in environmental ambience. We report that the answer to both questions is 'no'. Eating and fear generated by GABAergic inhibition of accumbens shell does not need endogenous dopamine. Also, the appetitive/fearful valence generated by GABAergic muscimol microinjections resists environmental retuning and is determined almost purely by rostrocaudal anatomical placement. These results suggest that nucleus accumbens GABAergic release of fear and eating are relatively independent of modulatory dopamine signals, and more anatomically pre-determined in valence balance than release of the same intense behaviors by glutamate disruptions.
\end{abstract}

\section{Introduction}

Local inhibitions in medial shell of the nucleus accumbens (NAc) of the rat produce intense appetitive and/or fearful motivated behaviors organized along a rostrocaudal gradient resembling an 'affective keyboard'. That is, microinjections of either the AMPA ( $\alpha$-amino-3hydroxy-5-methylisoxazole-4-propionic acid) antagonist DNQX (6,7-dinitroquinoxaline-2,3-dione) or $\gamma$-aminobutyric acid (GABA) agonist muscimol induce intense appetitive behaviors such as eating (Maldonado-Irizarry et al., 1995; Stratford \& Kelley, 1997), especially at rostral sites in medial shell (Reynolds \& Berridge, 2001, 2002 , 2003). Induction of an appetitve psychological state is supported also by demonstrations that rostral muscimol microinjections induce conditioned place preference, and potentiate 'liking' reactions to taste (Reynolds \& Berridge, 2002; Faure et al., 2010). In contrast, progressively more caudal microinjections of these same drugs

Correspondence: Dr J. M. Richard, as above.

E-mail: jrichard@gallo.ucsf.edu

*Ernest Gallo Clinic and Research Center at UCSF, 5858 Horton Street, Suite 200, Emeryville, CA, 94608-2007, USA

Received 10 December 2012, revised 5 February 2013, accepted 20 February 2013 produce increasingly fearful or aversive behaviors, including distress vocalizations and escape attempts elicited by the experimenter's touch, conditioned place avoidance, and spontaneous emission of defensive treading/burying behavior, an anti-predator response which rodents use to throw debris at a localized threat (i.e. rattlesnake or shock prod) (Coss \& Owings, 1978; Treit et al., 1981; Reynolds \& Berridge, 2001, 2002, 2003; Faure et al., 2010). Microinjections at intermediate locations produce graded mixtures of appetitive and fearful behaviors.

While keyboard patterns of intense motivations are generated by both methods of inhibition, GABAergic vs. glutamatergic effects differ in important respects. For example, while GABAergic muscimol microinjection amplifies positive hedonic 'liking' reactions to tastes at rostral sites and conversely amplifies negative disgust reactions at caudal sites (Reynolds \& Berridge, 2002; Faure et al., 2010), glutamate disruption fails to change affective 'liking' or disgust reactions, even when DNQX microinjection generates comparably intense levels of appetitive/fearful motivated behaviors (Faure et al., 2010).

Here we were interested in comparing motivations generated by GABAergic vs. glutamatergic NAc inhibitions on two other features. One feature is that endogenous local dopamine is necessary for glu- 
tamate disruptions to generate either desire or dread. That is, mixing dopamine antagonists into a DNQX microinjection prevents generation of intense eating and fear (Faure et al., 2008; Richard \& Berridge, 2011). However, given that the GABAergic inhibition generates changes in hedonic impact as well as motivation, and that dopamine does not mediate the hedonic impact of tastes (Peciña et al., 1997, 2003; Berridge \& Robinson, 1998), one might infer that the GABAergic inhibition should not require dopamine to generate intense motivations. Is GABAergic generation of eating and fear independent of local dopamine?

Another feature of the glutamate keyboard is that the ratio of appetitive to fearful behaviors is retuned by current environmental ambience, especially at intermediate rostrocaudal 'keys'. In a familiar and comfortable home environment, DNQX microinjections at more than $80 \%$ of sites generate positive appetitive behavior while the fear-generating zone shrinks to a far-caudal $20 \%$; by contrast, in a stressfully loud and bright environment DNQX instead generates mostly fearful behavior at $80 \%$ of sites (Reynolds \& Berridge, 2008; Richard \& Berridge, 2011). Therefore, we also asked: Is GABAergic generation of appetitive and fearful motivations similarly retuned by environmental ambience?

\section{Materials and methods}

\section{Experimental design}

We assessed appetitive and fearful behavior generated by GABAergic inhibition via muscimol microinjections to assess the contribution of endogenous dopamine signaling, and to detect any influence of changes in emotional ambience of the environment. We assessed the role of endogenous dopamine by combining a D1 antagonist and $\mathrm{D} 2$ antagonist in the same microinjection with the $\mathrm{GABA}_{\mathrm{A}}$ agonist muscimol. We separately assessed the influence of emotional ambience by comparing motivations generated by muscimol microinjections or DNQX microinjections in three different environments: 'Stressful' (noisy and bright), 'Standard' lab and 'Home' (a rat's own home-room: dark, quiet, familiar odor). To estimate the area of functional drug spread and to assess the neurobiological impact of dopamine blockade on GABAergic neuronal suppression, a Fos plume analysis was conducted on a separate group of rats.

\section{Subjects}

Male Sprague-Dawley rats $(280-400 \mathrm{~g}$, total $n=95$; dopamine dependence group, $n=18$; environment shift groups, $n=59$; Fos Plume group, $n=18$ ) from an in-house breeding colony were housed on a reversed 12:12-h light-dark cycle at $\sim 21{ }^{\circ} \mathrm{C}$ with ad libitum access to both food and water. All experimental procedures were approved by the University Committee on the Use and Care of Animals at the University of Michigan and were carried out in accordance with the guidelines on animal care and use of the National Institutes of Health of the United States.

\section{Cranial cannulation surgery}

All rats were anesthetized using a mixture of ketamine $(80 \mathrm{mg} / \mathrm{kg})$ and xylazine $(5 \mathrm{mg} / \mathrm{kg})$ and pretreated with atropine $(0.05 \mathrm{mg} / \mathrm{kg})$ to prevent respiratory distress. After anesthesia induction, rats were placed in a stereotaxic apparatus (David Kopf Instruments, Tujunga, CA, USA), with the mouth bar set to $5.0 \mathrm{~mm}$ above intra-aural zero, so that cannulae could be angled to avoid puncturing the lateral ventricles. Bilateral stainless steel cannulae $(14 \mathrm{~mm}, 23$ gauge) were aimed $2 \mathrm{~mm}$ above points throughout the rostrocaudal extent of the medial shell, between coordinates anteroposterior (AP) +2.4 to $+3.4 \mathrm{~mm}$ ahead of bregma, mediolateral (ML) $\pm 1.0 \mathrm{~mm}$ from the midline, and dorsoventral (DV), +5.7-6.0 mm below skull. Cannulae were anchored to the skull using surgical screws and secured with dental cement; stainless steel obturators (28 gauge) were insert to prevent occlusion of the cannulae. Post-surgery, rats received cefazolin $(75 \mathrm{mg} / \mathrm{kg})$ to prevent infection and carprofen $(5 \mathrm{mg} / \mathrm{kg})$ for analgesia. Rats were allowed to recover for 7 days before testing.

\section{Intracerebral microinjections}

Drug microinjections were always administered bilaterally in a 0.5 $\mu \mathrm{L}$ volume on test days spaced at least $48 \mathrm{~h}$ apart. On test days, solutions were brought to room temperature $\left(\sim 21{ }^{\circ} \mathrm{C}\right)$, inspected to confirm the absence of precipitation, and infused at a speed of $0.3 \mu \mathrm{L} / \mathrm{min}$ using a syringe pump attached via PE-20 tubing to stainless steel injectors (16 mm, 29 gauge) which extended $2 \mathrm{~mm}$ beyond the end of the guide cannulae into medial shell. Injectors were left in place for $1 \mathrm{~min}$, and then obturators were replaced and rats were immediately placed in one of the testing chambers described below.

\section{GABA/dopamine interaction experiment}

For GABAergic muscimol microinjections in medial shell, rats $(n=18)$ received a dose of muscimol $(75 \mathrm{ng} / 0.5 \mu \mathrm{L}$ per side) previously shown to generate intense eating and fear (Faure et al., 2010). Muscimol was microinjected either alone or, to test the role of endogenous dopamine, in combination with two dopamine D1 and D2 antagonists as a mixture in the same microinjection: the selective D1 antagonist SCH23390 $(R(+)-7$-chloro-8-hydroxy-3-methyl 1-phenyl-2,3,4,5,-tetrahydro-1H-3-benzazepine) at a dose of $3 \mu \mathrm{g} / 0.5 \mu \mathrm{L}$ per side, plus the selective D2 antagonist raclopride (3,5-dichloro$N$-\{[(2S)-1-ethylpyrrolidin-2-yl]methyl $\}$-2-hydroxy-6-methoxybenzamide) at a dose of $5 \mu \mathrm{g} / 0.5 \mu \mathrm{L}$ per side. These doses of dopamine antagonists have been shown previously to prevent DNQX microinjections in NAc shell from producing eating and fearful behaviors (Faure et al., 2008). Each rat received four microinjections in balanced order: (i) vehicle alone (artificial cerebrospinal fluid, ACSF), (ii) muscimol alone, (iii) dopamine antagonists alone (D1 \& D2 dopamine antagonists without muscimol) and (iv) muscimol plus D1 \& D2 dopamine antagonists. Immediately after every microinjection the rat was placed in a chamber in a conventional laboratory testing room with normal daylight illumination conditions (white fluorescent light intensity 550-650 lux) and moderate intensity ambient noise (65-70 dB).

\section{GABA vs. glutamate environmental shift experiment}

To compare environmental ambience modulation of intense motivations produced by glutamate disruption or GABA inhibition, each rat was tested in three different emotional environments either after DNQX or vehicle microinjections (six tests), or after muscimol or vehicle microinjection (six tests). DNQX was microinjected at a dose of $450 \mathrm{ng} / 0.5 \mu \mathrm{L}$ per side and muscimol was injected at a dose of $75 \mathrm{ng} / 0.5 \mu \mathrm{L}$ per side, doses which were selected to produce roughly equal levels of motivated behaviors from previous studies (Faure et al., 2010). Some rats $(n=29)$ received microinjections of either DNQX or its vehicle (50\% DMSO and $50 \% 0.15 \mathrm{~m}$ saline) on separate days, prior to being tested twice each in the Home, Stressful and Standard environments, for a total of six test days and 
six microinjections per rat. Other rats $(n=30)$ received microinjections of either muscimol or its vehicle (ACSF) prior to being tested twice in each of the three environments, also for a total of six test days. The order of drug and environment conditions was counterbalanced in each group.

The Standard lab environment was identical to test conditions described above, used in most previous studies and for the dopamine blockade experiment (standard lab room, fluorescent lighting, low to moderate levels of ambient noise). The Home environment test occurred in the rats' own home-room, and was characterized by dim red lighting (5-10 lux), the familiar odors of the housing room, and low levels of ambient noise primarily consisting of sound from the ventilation systems $(65-70 \mathrm{~dB})$ and from other rats. The Stressful environment was conducted in an unfamiliar lab room, featuring high-intensity sensory-stimulation of light and sound. Extra light was provided by additional incandescent lamps that were directed at the transparent test chamber (1000-1300 lux within the chamber). Loud, unpredictable sound was presented continuously through the test [raucous rock music from the continuous full-album soundtrack of Raw Power by Iggy \& The Stooges (1973; Iggy Pop reissue 1997); 80-86 dB]. In previous preference tests, rats have been shown to prefer the Home environment condition over the Standard environment condition and to prefer the Standard lab environment over the Stressful condition (Reynolds \& Berridge, 2008).

\section{Behavioral tests of unconditioned motivated behaviors}

Prior to the first test day, rats were habituated to handling and procedures for 7 days: rats were handled for $10 \mathrm{~min}$ per day for 3 days, and then were habituated to the testing procedure and apparatus for $1 \mathrm{~h}$ each on four additional days. On the 4th day of habituation rats received 'mock' microinjections of vehicle before being placed in the chamber. On drug test days, rats received one of the microinjections described above and were placed immediately in a transparent testing chamber $(23 \times 20 \times 45 \mathrm{~cm})$ which contained pre-weighed food ( $20 \mathrm{~g}$ rats chow) and ad libitum water. The chamber always contained granular cob bedding $(\sim 3 \mathrm{~cm}$ deep) to allow expression of defensive treading behavior. Rats remained in the chamber for $60 \mathrm{~min}$ while behavior was videorecorded, to be coded later for analysis.

At the end of each session, rats were removed by the experimenter's gloved hand using a standardized slow-approach hand motion in order to quantify any fearful distress calls, escape attempts or defensive bites elicited by human touch. Following a $\sim 5 \mathrm{~s}$ approach towards the testing cage, the experimenter slowly reached towards the rat, taking $\sim 2 \mathrm{~s}$. Upon contact, the experimenter lightly brushed the side of the rat with gloved fingertips, taking $\sim 1 \mathrm{~s}$, before lifting the rat from the chamber in a gentle movement that lasted $\sim 2 \mathrm{~s}$. The observer recorded any (i) attempts by the rat to escape when touched (e.g. frantic jumps or runs away from the hand), (ii) bites or attempts to bite the gloved hand and (iii) audible distress vocalizations elicited by the approaching hand.

\section{Behavioral coding of videorecorded behaviors}

Videorecorded behaviors were scored offline by observers blind to drug conditions. Incidences of elicited fearful distress vocalizations, escape dashes and bite attempts directed at the experimenter's hand were scored when the rat was gently picked up at end of the test session (Reynolds \& Berridge, 2003), at which time the total weight of chow pellets consumed was also recorded. Behaviors emitted spontaneously and videotaped during the 1-h test were subsequently scored by experimenters blind to treatment for the total cumulative duration (seconds) for each of the following: eating behavior (involving both appetitive approach and voluntary initiation of ingestion plus consummatory chewing and swallowing of food), drinking behaviors (licking from water spout), fearful defensive treading/burying behavior (defined as active spraying or pushing of bedding with rapid alternating thrusts of the forepaws, spatially directed generally towards the brightly lit front or corners of the cage) and grooming behavior (a stereotyped sequence described in Aldridge et al., 1993). Observers scored the total number for behaviors which tended to occur as discrete events, including appetitive behaviors such as food carrying (transportation of food pellets in the mouth) and food sniffs (sniffing near the food for at least $1 \mathrm{~s}$ ), and two general motor activities: rearing (forepaws at least one inch off the floor) and cage crosses (forepaws and head cross the halfway point of the cage)

\section{Histology}

Following behavioral testing rats were killed with an overdose of sodium pentobarbital, and their brains were removed and fixed in $10 \%$ paraformaldehyde for 1-2 days and in $25 \%$ sucrose solution for 3 days. To assess microinjection site locations, brains were sliced at $60 \mu \mathrm{m}$ on a freezing microtome, and stained with Cresyl violet. Microinjection sites were mapped onto coronal slices from a rat brain atlas (Paxinos \& Watson, 2007), which were then used to extrapolate the position of each site on a sagittal slice. This allowed for the presentation on the same maps of most of the rostrocaudal and dorsoventral extent of medial shell. Functional effects on appetitive and fearful behaviors were mapped using color-coding to express intensity of changes in motivated behaviors for individual behaviorally tested rats. Symbols were sized to match the maximal diameter of Fos plumes as found here and previously (Faure et al., 2010; Richard \& Berridge, 2011). For rostral vs. caudal statistical comparisons, sites were classified as rostral shell if they were located more than $1.4 \mathrm{~mm}$ ahead of bregma, and as caudal if they fell behind this benchmark.

\section{Fos-like protein immunohistochemistry}

To map the size of the area where microinjections were likely to have impacted local tissue, as well as to assess the neurobiological effects of dopamine antagonists on GABA inhibition with muscimol, we conducted a Fos plume analysis on a separate group of rats after a single microinjection to detect maximal spread of neural impact. This was important because we have previously shown that the intensity and diameter of changes in Fos expression are suppressed following several DNQX microinjections (Richard \& Berridge, 2011). That finding indicates maximal neural impact spread may best be assessed after a single microinjection (Richard \& Berridge, 2011), just as behavioral efficacy of microinjections may be strongest initially (Kelley et al., 2000). Therefore, we analysed Fos after only a single microinjection to avoid potential mapping problems due to underestimation of impact spread.

Fos plumes are used specifically as a measure of diameter for the spread of neural impact locally surrounding a drug microinjection. No assumptions are required that Fos-expressing neurons are identical to receptor-bearing neurons (local neurons that are indirectly modulated via synaptic recruitment cannot be excluded from contributing to behavioral effects of a microinjection, so are also included in measures of impact spread), or that Fos-expressing neurons are those functionally responsible for generating an observed 
behavior. Nor do we assume that Fos elevation necessarily signifies neuronal depolarization or that Fos inhibition signifies hyperpolarization (Dragunow \& Faull, 1989). Information regarding the diameter of Fos plumes is useful for identifying how far the immediate neural impact of a drug microinjection spreads from its center. The clear spatial cut-off of local continguous Fos plumes allows local impact spread to be easily distinguished from circuit-level Fos changes at more distant sites, which are typically separated by unaffected spaces with no change in Fos expression extending up to several millimeters from the site. Our study used Fos plumes to estimate maximal spread of local impact. To construct maps for localization of function, we combined that information about spread diameter with functional information about the behavioral consequences of drug microinjections at each mapped site.

Rats used for Fos analysis $(n=18)$ were anesthetized with an overdose of sodium pentobarbital and transcardially perfused $90 \mathrm{~min}$ after bilateral microinjection of (i) vehicle in NAc $(n=5)$, (ii) muscimol $(n=6)$, (iii) dopamine antagonists $(n=3)$ or (iv) mixture $(n=3)$ or (v) no injection $(n=1)$ for Fos plumes analysis. Brains were removed and placed in $4 \%$ paraformaldehyde for $4-24 \mathrm{~h}$, and then transferred to $25 \%$ sucrose (in $0.1 \mathrm{~m}$ sodium phosphate buffer) for 3 days. Brains were sliced at $40 \mu \mathrm{m}$ on a freezing microtome, and processed for Fos-like immunoreactivity using NDS, goat anticfos (Santa Cruz Biotechnology, Santa Cruz, CA, USA) and donkey anti-goat Alexa Fluor 488 (Invitrogen, Carlsbad, CA, USA) as described previously (Faure et al., 2008, 2010; Reynolds \& Berridge, 2008). Sections were mounted, air-dried and coverslipped with ProLong Gold antifade reagent (Invitrogen).

\section{Fos plume analysis}

Immunoreactivity for Fos-like protein was visualized using a Leica microscope equipped for fluorescent microscopy, using a filter with an excitation band at 480-505 $\mathrm{nm}$ for Fos-positive cells and images were taken using MCID Core software. For analysis of drug spread, Fos plume images were taken in the areas surrounding the microinjection with the most intense areas of Fos expression, just caudal to the end of the injector tip, surrounding a small focal point of necrosis. Fos-labeled cells were individually counted within successive blocks $(50 \times 50 \mu \mathrm{m})$, along eight radial arms emanating from the center of the necrosis, with $10 \times$ magnification (Fig. 1). Zones of Fos elevation (or 'plumes') were assessed as described previously for muscimol and dopamine antagonist microinjections (Reynolds \& Berridge, 2008). Fos plume estimates of the diameter of DNQX microinjection impact were adopted from a previous study that used the same dose and volume of DNQX microinjections in NAc medial shell (Richard \& Berridge, 2011).

\section{Statistical analysis}

The effects of dopamine blockade on muscimol-induced motivated behaviors were assessed using a three-factor mixed within- and between-subject ANOVA [muscimol $\times$ dopamine blockade $\times$ anatomical level (rostral vs. caudal)]. When significant effects were found, rats were split into rostral and caudal groups and additional two-way (muscimol $\times$ dopamine blockade) and one-way ANovas (main effect of drug) were conducted, including pairwise comparisons with Sidak corrections. For analysis of binomial data (vocalizations, escape attempts and bite attempts) Cochran's Q and McNemar's repeated measures tests were used. The effects of DNQX or muscimol in the three different emotional environments were compared using a three-factor mixed within- and between-subjects ANOVA [drug $\times$ anatomical level (rostral vs. caudal) $\times$ environment (Stressful vs. Standard vs. Home)] for all parametric behaviors. When significant effects were found, rats were split into rostral and caudal groups and additional one-way ANOvas were conducted on the difference between vehicle and drug between the three environments, including pairwise comparisons with Sidak corrections. We also assessed the percentage of rats which met criteria for appetitive, defensive or mixed valence behaviors, and tested whether that changed between the three environments for DNQX vs. muscimol, using Cochran's Q and McNemar's repeated-measures test, and whether the proportion of rats that switched differed between DNQX and muscimol using Pearson's chi-squared test.

\section{Results}

\section{Dopamine blockade experiment: dopamine is not needed for GABAergic motivation}

Local blockade of dopamine D1 and D2 receptors failed to impair the ability of GABAergic muscimol microinjections to generate intense levels of motivated behaviors (5- to 75-times above vehicle control levels). The generation of neither appetitive eating nor fearful defense/escape behaviors was prevented by the addition of dopamine antagonists to the muscimol microinjection, and muscimolgenerated levels always remained high above vehicle levels regardless of whether dopamine antagonists were present or absent (Figs 1 and 2). In rostral shell, muscimol microinjections generated robust tripling of food intake and of time spent eating, with or without dopamine antagonists (Figs $1 \mathrm{~A}$ and $\mathrm{B}$, and $2 \mathrm{~B}$; eating, interaction of muscimol by placement, $\left.F_{1,14}=14.51, P=0.002\right)$. The addition of dopamine antagonists did not reduce these intense appetitive behaviors generated at rostral sites, and eating remained intense, at more than double vehicle levels (Figs 1A and 2A; rostral shell, eating, interaction of muscimol and D1/D2 antagonists, $F_{1,8}=<0.01$, $P=0.996$ ). By comparison, caudal shell sites of muscimol microinjection with or without dopamine antagonists generated a 60-fold increase in defensive treading behavior (Figs $1 \mathrm{C}$ and $2 \mathrm{~B}$; interaction of muscimol by placement, $F_{1,14}=6.15, P=0.026$ ), as well as distress vocalizations, escape attempts and sometimes bite attempts in response to the experimenter's approach and touch after the treading test (Figs 1D and 2C; vocalizations and escape attempts, $P<0.001)$. Dopamine antagonists did not alter the level of fearful defensive treading generated by muscimol at caudal sites, which remained at more than 50-fold vehicle levels (Figs $1 \mathrm{C}$ and $2 \mathrm{~B}$; interaction of muscimol and D1/D2 antagonists, $F_{1,6}=0.86, P=0.389$ ). Dopamine blockade also had no effect on caudal distress vocalizations, escape attempts or bite attempts observed after the mixture of muscimol and dopamine antagonists when compared with muscimol alone (Figs 1D and 2C; McNemar's test, vocalizations, $P=0.375$; escape attempts, $P=0.180$; bite attempts, $P=1.000$ ).

The persistence of intense GABAergic eating and fear after local dopamine blockade contrasts with the dramatic reduction of motivated behaviors generated by corticolimbic glutamate disruptions we previously reported to be caused by similar dopamine blockade (Faure et al., 2008; Richard \& Berridge, 2011). However, by themselves, microinjection of dopamine antagonists here (without muscimol) did suppress lower spontaneous baseline levels of appetitive eating and food intake below vehicle to nearly zero (Figs $1 \mathrm{~A}$ and B; time spent eating, general effect of D1/D2 antagonists, $F_{1,15}=7.853, P=0.014$; food intake grams consumed, average of $0.25 \pm 0.68 \mathrm{~g}$ under D1/D2 antagonists vs. $1.65 \pm 1.28 \mathrm{~g}$ under vehicle; food intake, general effect of D1/D2 antagonists, $F_{1,15}=8.674, P=0.012$ ). 

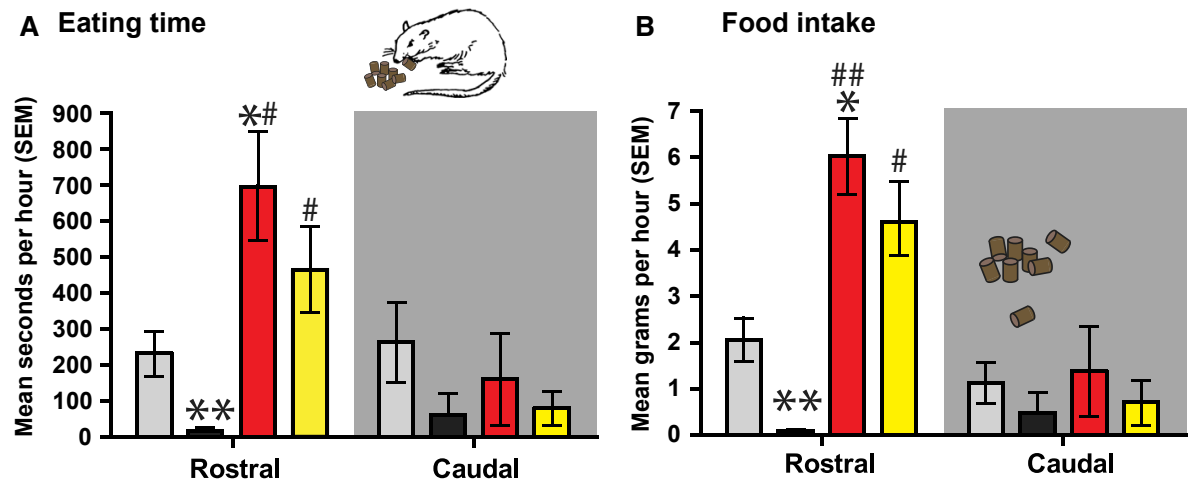

\section{Fearful treading}

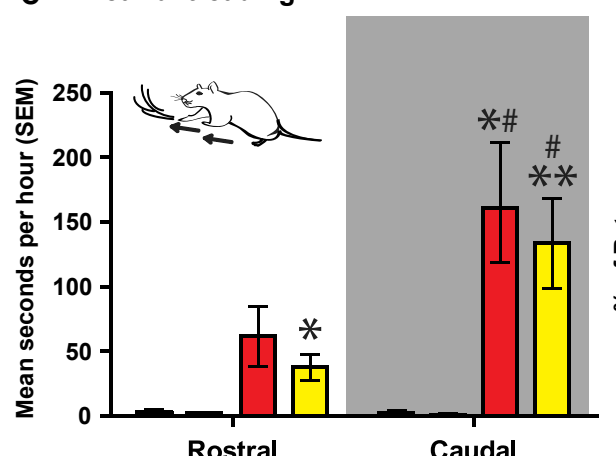

\section{Distress vocalizations}

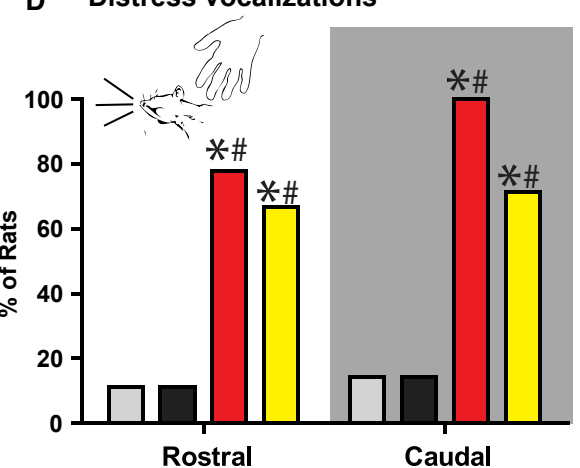

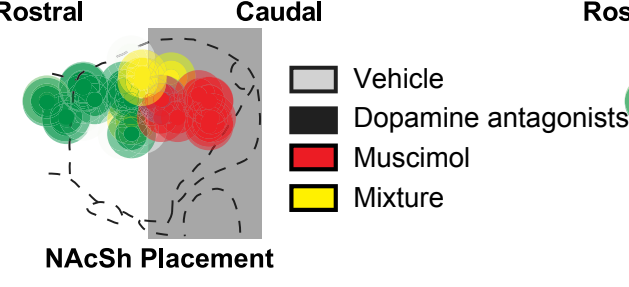

NAcSh Placement

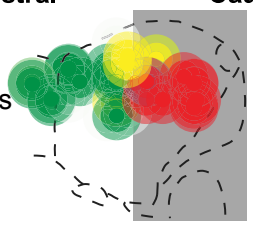

NAcSh Placement

FIG. 1. Summary graphs of the effect of local dopamine blockade on muscimol-generated eating and fearful behaviors. Time spent eating (A), amount of food intake (B), time spent defensive treading/burying (C), as well as incidence of distress vocalizations in response to human touch (D), elicited by vehicle (grey), dopamine antagonist combination of raclopride and SCH23390 (black), muscimol (red) and mixture of muscimol and dopamine antagonist (yellow) in rostral $(n=9)$ and caudal $(n=7)$ regions of medial NAc shell. Errors bars indicate SEM; ${ }^{*} P<0.05, * * P<0.01$ change from vehicle, ${ }^{\#} P<0.05,{ }^{\# \#} P<0.01$ change from dopamine antagonists alone, pairwise comparisons using Sidak corrections for multiple comparisons (eating, food intake and defensive treading) or McNemar's test (distress vocalizations). Muscimol alone and mixture were never significantly different. Summary maps below show the localization of microinjections that produced primarily appetitive (green symbols), defensive (red symbols) or mixed appetitive and defensive (yellow symbols) in rostral (white) vs. caudal (grey) shell.

\section{Appetitive vs. fearful motivational valence generated by GABAergic inhibition is purely anatomically determined and resists change by environmental ambience}

Muscimol microinjections in NAc shell generated the same rostrocaudal keyboard-like pattern of intense eating, fearful and mixed valence behaviors across all three environments, resisting environmental retuning (Figs 3 and 4). At rostral sites in medial shell muscimol generated intense appetitive behavior equally in Home, Standard and Stressful environments (main effect on eating, $F_{1,27}=6.309, P=0.018$; interaction with placement, $F_{2,27}=5.672$, $P=0.009)$. At more caudal locations in medial shell, muscimol generated intense defensive behaviors in all three environments (main effect on treading, $F_{1,27}=4.511, P=0.043$; interaction with placement, $F_{2,27}=6.388, P<0.005$; Fig. 5), such that at least $46 \%$ of rats emitted distress vocalizations to the experimenter, and at least $21 \%$ of rats attempted to escape in each environment (McNemar's tests, vocalizations: Standard, $P=0.000$, Stressful, $P=0.004$, Home $P=0.000$; escape attempts: Standard, $P=0.031$, Stressful, $P=0.008$, Home, $P=0.031$; Fig. 6). Switching between environments had no detectable influence on the intensity of muscimol-elic- ited appetitive eating at any site (muscimol by environment: eating, $F_{4,54}=1.874, P=0.128$; Fig. 7 ) and did not alter the size of the appetitive zone in which eating was elicited (Cochran's Q $(2)=0.400, P=0.819)$. Environmental changes also did not alter fearful escape attempts or distress vocalizations elicited by the approach of the experimenter's gloved hand (vocalizations, Cochran's $\mathrm{Q}(2)=0.500, \quad P=0.779$; escape attempts, Cochran's Q $(2)=0.727, P=0.695)$, and altered only one category of fearful reaction: spontaneous defensive treading.

The only environmental modulation effect observed was that the dark, quiet and familiar Home environment virtually eliminated spontaneous emission of defensive treading $\left(F_{1,26}=8.551\right.$, $P<0.007$; McNemar's tests, vs. Standard, $P=0.005$; vs. Stressful, $P=0.002$; Fig. 5). However, the noisy and bright Stressful environment did not increase defensive treading behavior over the already intense levels produced by caudal muscimol microinjections in the Standard environment (muscimol by environment: $F_{1,26}=0.163$, $P=0.690$ ), and failed to switch any rostral sites from generating purely appetitive eating to defensive treading (Stressful vs. Standard, McNemar's test, $P=1.000$ ). Also, the reactive and even more extreme threat-evoked defensive reactions of distress vocalizations 

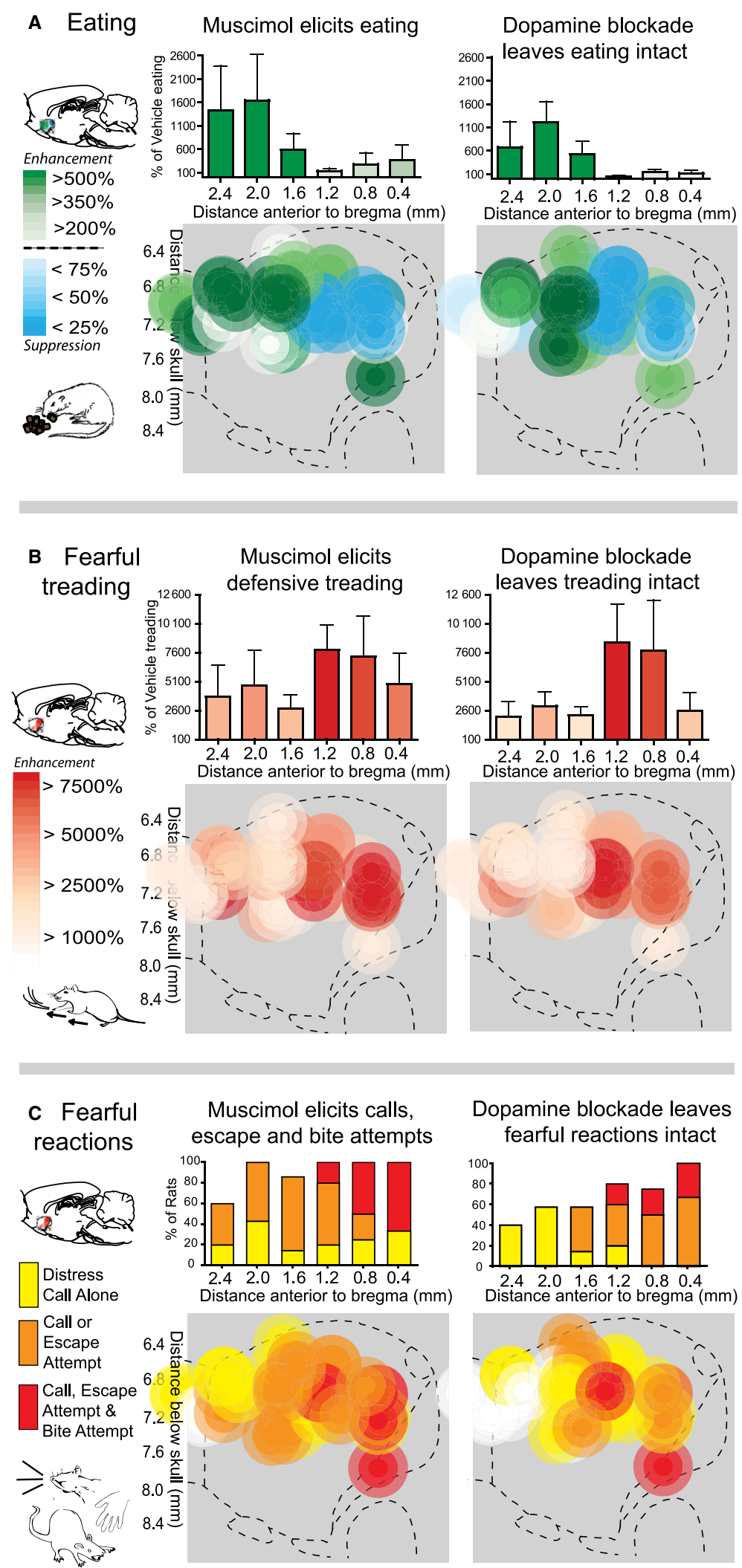

FIG. 2. Effects of dopamine antagonism on muscimol-induced eating and defensive fearful behaviors. Fos plume maps $(n=16)$ of the generation in medial shell of eating (A), defensive treading (B), and fearful calls, escape attempts and bite attempts (C) by muscimol (left) and a mixture of muscimol plus dopamine antagonists (right). Local dopamine blockade failed to prevent muscimol-generated eating or fearful behaviors, despite its previously reported ability to prevent DNQX-induced eating and fear, and its generally suppressive effects. Histograms bars below the maps show behavior as a percentage of vehicle (eating, A; treading, B) or percentage of subjects (calls, escape attempts and bite attempts, C) for each behavior at rostrocaudal level as marked along the medial shell $($ error bars $=\mathrm{SEM})$. 


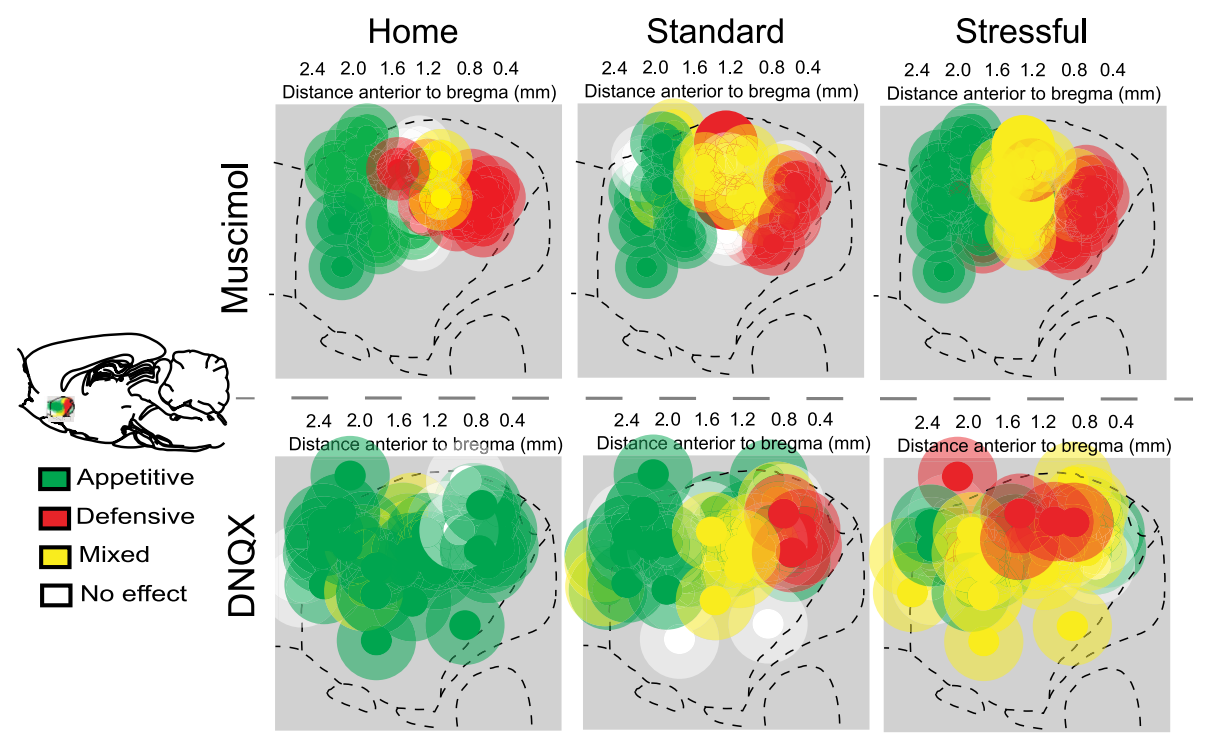

FIG. 3. Summary maps of behavior. Summary maps (A) show behavior produced by muscimol (top) or DNQX (bottom) in either the Home (left), Standard (middle) or Stressful (right) environments. Each subject $(n=59)$ was designated as producing primarily appetitive (green symbols), defensive (red symbols) or mixed appetitive and defensive (yellow symbols) motivated behavior following DNQX microinjection. In a Standard environment, purely appetitive eating behavior and food intake (criteria for including a site was a $>200 \%$ increase in eating) was primarily stimulated in rostral shell by DNQX and muscimol. Fearful distress calls, escape attempts and spontaneous emission of defensive treading-burying (criteria for including a site was a $>500 \%$ increase in treading over vehicle levels, or emission of a defensive reaction to the experimenter) were primarily stimulated in caudal shell by DNQX. Mixed sites met criteria for both motivations. Testing in the Stressful environment shifted fearful behavior produced by DNQX into more rostral regions, whereas testing in the Home environment virtually eliminated all defensive behavior produced by DNQX. Muscimol produced a similar rostrocaudal gradient of eating and fear regardless of environment.

and escape attempts that were elicited by the experimenter's touch remained equally intense and unchanged across all three environments after caudal muscimol microinjections (distress vocalizations, Cochran's $\mathrm{Q}(2)=0.500, P=0.779$; escape attempts, Cochran's Q $(2)=0.727, P=0.695$; Fig. 6).

Thus, the overall pattern of rostral shell generation of intense eating, middle generation of mixed eating/fear and caudal generation of intense fear appeared stable, and determined purely by anatomical location of each GABAergic microinjection that induced local hyperpolarization. The valence tuning of individual microinjection sites rarely shifted across environments. Only the behavioral intensity of spontaneous defensive treading for middle and caudal sites was at all influenced by environment, and only by being dampened in the presence of the familiar and comfortable Home conditions. All other aspects of intense motivated behaviors generated by muscimol microinjections were invulnerable to environmental changes.

By contrast to GABAergic inhibition, the keyboard pattern generated by DNQX microinjections was powerfully shifted both anatomically and in behavioral intensity generated at particular sites by changes in environmental ambience, as expected (Figs 3 and 4A; interaction with environment: eating, $F_{2,44}=7.359, P=0.002$; treading, $F_{2,44}=4.985, P=0.011$; distress vocalizations, Cochran's $\mathrm{Q}(2)=11.556, \quad P=0.003 ;$ escape attempts, Cochran's $\mathrm{Q}(2)=$ 6.500, $P=0.039$ ). The Stressful environment nearly doubled the size of the medial shell zone where defensive treading was generated, in comparison with the Standard environment, to more than $55 \%$ of sites (vs. $31 \%$ in Standard), more than quadrupling the defensive zone in comparison with the Home environment (overall effect of environment on the percentage defensive sites, Cochran's $\mathrm{Q}(2)=10.391, P=0.006$; McNemar's test, $P=0.002$, Fig. 5). Likewise, the Stressful environment doubled the zone in which DNQX produced attempts to escape from the experimenter's hand, compared with the Standard environment (to $7 \%$ of sites; $\mathrm{McNe}$ mar's tests, Lab vs. Stressful, $P=0.25$; Home vs. Stressful, $P=0.125$; Fig. 6) and quadrupled the zone where DNQX produced reactive distress vocalizations (to 28\%; McNemar's tests, Lab vs. Stressful, $P=0.070$; Home vs. Stressful, $P=0.008$ ).

Conversely, the familiar Home environment nearly eliminated the fearful caudal zone where DNQX generated spontaneous defensive treading behavior (Cochran's $\mathrm{Q}(2)=10.391, P=0.006$; difference between Stressful and Home environments, McNemar's test, $P=0.002$, Fig. 5), and completely eliminated generation of reactive responses such as distress vocalizations and escape attempts to the experimenter's hand (McNemar's test, Lab vs. Home, $P=0.500$, Stressful vs. Home, $P=0.008$; Fig. 6). At the same time, the Home environment expanded the 'appetitive zone' where DNQX produced purely appetitive behavior to more than $75 \%$ of medial shell (vs. $31 \%$ in the Stressful environment; Fig. 3), consistent with previous findings (Reynolds \& Berridge, 2008). Testing the Stressful environment slightly, but non-significantly, reduced the intensity of DNQXinduced eating (DNQX by environment, $F_{1,23}=3.404, P=0.078$; Fig. 7).

\section{Fos plume analysis of muscimol and dopamine antagonist microinjections}

Mapping of effects was aided by measurements of drug impact spread for muscimol microinjections, as reflected in Fos plumes of about $0.5 \mathrm{~mm}$ in diameter. Microinjections of muscimol alone in NAc medial shell altered Fos expression in an area with a total radius of $0.24 \mathrm{~mm}$ : containing a small 0.15 -mm-radius excitatory plume center (volume $\left.=0.014 \mathrm{~mm}^{3}\right)$, of elevated Fos expression $(150 \%$ of vehicle levels), encompassed by a larger of 0.24-mm-radius (volume $=0.06 \mathrm{~mm}^{3}$ ) inhibitory or 'anti-plume' surround (Fos reduced to $<75 \%$ of vehicle levels; Fig. 8 ), similar to our previous reports 


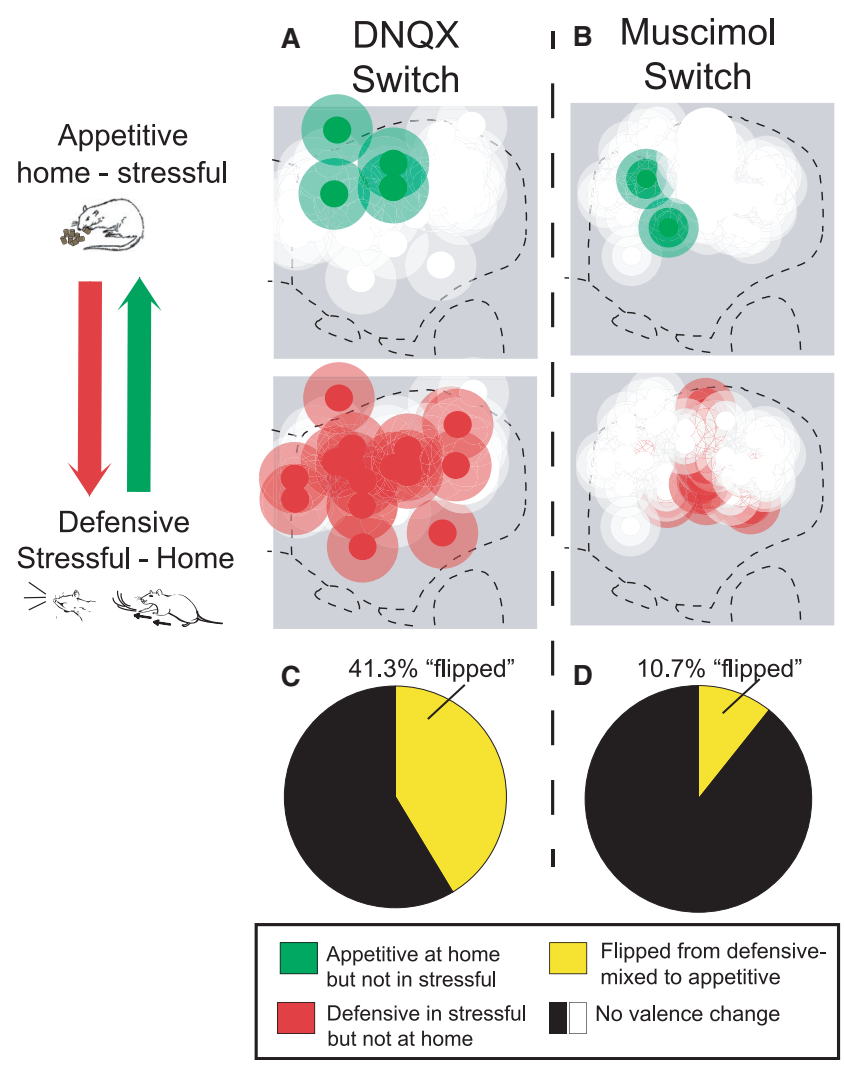

FIG. 4. Maps of changes in motivational valence between the Home and Stressful environment. Summary maps show sites where DNQX (A) or muscimol (B) generated either intense eating behavior (top; green; $>200 \%$ of vehicle) in the Home environment but not in the Stress environment, or intense defensive behavior (bottom; red; $>500 \%$ of vehicle level treading or defensive reaction to the experimenter) in the Stressful environment but not in the Home environment. Sites mapped in white produced the same valence of behavior in both the Home and the Stressful environment. The criterion for designating a site as appetitive was a $>200 \%$ increase in eating; criteria for designating a site as defensive was a $>500 \%$ increase in treading over vehicle levels or emission of a defensive reaction to the experimenter. The percentage of rats that 'flipped' between mainly defensive/mixed in the Stressful environment and purely appetitive in the Home environment was significantly greater in rats that received DNQX (C) than rats that received muscimol (D), and the number of rats that 'flipped' when given muscimol was not significant.

(Faure et al., 2010). Dopamine D1 and D2 antagonists, combined together but without muscimol, produced a tiny excitatory center (volume $=0.005 \mathrm{~mm}^{3}$ ) of $150 \%$ of vehicle-level Fos expression up to $0.10 \mathrm{~mm}$ from the microinjection center, surrounded by a very large 0.41-mm-radius robust inhibitory 'anti-plume'. The anti-plume contained a dense inhibitory middle layer of $0.16 \mathrm{~mm}$ radius (volume $\left.=0.018 \mathrm{~mm}^{3}\right)$ of intense Fos suppression $(<50 \%$ of vehicle levels), contained within a larger 0.41-mm-radius (volume $=0.29 \mathrm{~mm}^{3}$ ) but less intense anti-plume of moderate Fos suppression (50-75\% of vehicle levels), consistent with previous reports (Faure et al., 2008). Combining muscimol and dopamine antagonists in the same cocktail microinjection produced a similarly sized 0.4mm-radius anti-plume, containing an even larger 0.31-mm-radius middle layer of intense Fos suppression (volume $=0.135 \mathrm{~mm}^{3}$ ), surrounded by a thinner shell $(0.1 \mathrm{~mm}$ shell width; $0.4 \mathrm{~mm}$ total outer radius) of moderate Fos suppression (50-75\% of vehicle levels; total volume $=0.24 \mathrm{~mm}^{3}$ ). The diameter of estimated spread for DNQX microinjection was based on a previously reported total radius of $0.38 \mathrm{~mm}$ ( $\pm 0.05 \mathrm{~mm}, \mathrm{SEM})$ (Richard \& Berridge, 2011).

\section{Discussion}

GABAergic release of intense motivations by muscimol microinjection in NAc appears to be more anatomically predetermined by rostrocaudal location in medial shell than equivalent motivation generation by glutamate disruptions (Reynolds \& Berridge, 2008). GABAergic release of eating and fear is also more autonomous of endogenous dopamine inputs (Faure et al., 2008; Richard \& Berridge, 2011).

Here the addition of D1 (or D2) dopamine antagonists to GABAergic muscimol microinjections did not impair the generation of intense levels of eating and food intake at rostral sites nor of fearful spontaneous defensive treading behavior and of reactive frantic escape attempts and distress calls upon being touched at caudal sites. By contrast, addition of a D1 antagonist to a DNQX microinjection does block generation of either eating or fear by NAc glutamate disruption, and addition of a D2 antagonist additionally blocks generation of fear, as we previously showed (Richard \& Berridge, 2011).

Regarding environmental modulation, the valence of appetitive vs. fearful motivation generated by the GABAergic inhibition of NAc resisted re-tuning by ambience changes. Muscimol microinjections always released anatomically determined mixtures of intense motivations, based solely on keyboard position along the rostrocaudal axis of medial shell, regardless of whether rats were exposed to the comfortable Home environment, the neutral Standard lab, or the loud and bright Stressful environment. By contrast, we confirmed that those environmental changes powerfully retuned the relative size of appetitive vs. fearful valence-generating zones produced by glutamate disruption.

The sole environmental retuning effect observed for GABAergic muscimol microinjections was that the dark Home environment nearly eliminated spontaneous defensive treading-burying, which in Standard and Stressful environments was directed specifically toward the front of the transparent cage. However, reactive distress calls and frantic escape jumps away from the experimenter's hand at the end of the session persisted in the dark Home environment after caudal muscimol microinjections, remaining unsuppressed, and those defensive escape reactions elicited by human touch arguably reflect even more intense fear than defensive treading. That pattern suggests that at best the Home environment suppresses moderate fear generation displayed in defensive treading-burying but does not suppress more intense fear generation displayed in reactive distress calls and frantic escape attempts (elicited by the potentially threatening stimulus of an approaching human hand). Additionally, an alternative explanation exists for why spontaneous treading was reduced: the dark Home environment (illuminated only by dim red light, which scotopic rat vision does not detect well) made it more difficult for rats to see visual stimuli toward which defensive treading is usually directed (sight of objects in the room beyond the cage, reflected light from curved corners of the cage). Thus, it may have been the removal of eliciting visual stimuli that was more responsible for reducing defensive treading, rather than suppression of fear. In the lighted Standard and Stressful environments, a rat typically threw bedding toward the reflecting corners or toward the transparent front wall of the chamber facing the room, often building a defensive mound of bedding between those stimuli and the rat (Reynolds \& Berridge, 2001). In the absence of those eliciting visual stimuli under darkness, conceivably even an actively fearful rat might not emit much spontaneous treading behavior, but could still react defensively to the multi-modal stimulus of an approaching hand. Especially considering that alternative explanation, and that all 


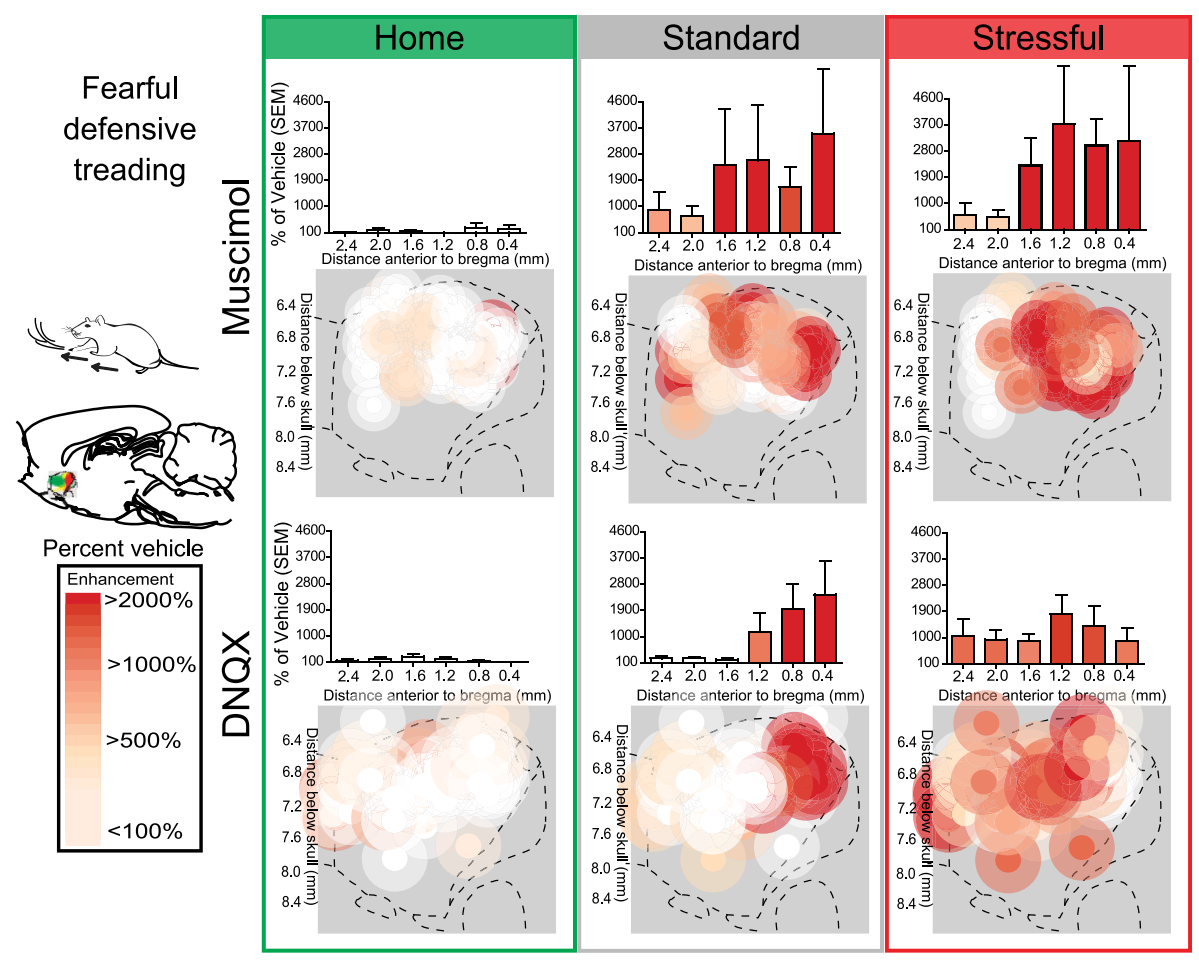

FIG. 5. Effect of changing environmental ambience on defensive treading produced by DNQX or muscimol. Fos plume maps $(n=59)$ of the generation of defensive treading by muscimol (top) or DNQX (bottom) in the Home (left), Standard (middle) or Stressful (right) environments. Testing in the Stressful environment had no effect on muscimol-generated treading, but the Home environment nearly eliminated muscimol-generated treading. This may be due to the removal of all visual cues that the animals usually tread toward. DNQX treading was produced at more rostral locations in a Stressful environment, but was nearly eliminated by testing in a Home environment. Histogram bars show treading as a percentage of vehicle at each rostrocaudal level as marked along the medial shell $($ error bars $=\mathrm{SEM})$.

other aspects of the rostrocaudal pattern of eating and fear generated by GABA inhibition remained intact across the three environments, we conclude that GABAergic muscimol generation of intense motivations is more robust, resistant to environmental retuning of valence and neuroanatomically pre-determined than similar motivations produced by glutamate disruption (Faure et al., 2008; Reynolds \& Berridge, 2008).

\section{Bases for subcortical autonomy}

Why should the GABAergic NAc keyboard for generating desire vs. dread be more anatomically pre-determined and autonomous of endogenous dopamine than the corresponding glutamatergic keyboard? One possible answer derives from the subcortical and intrinsic nature of endogenous GABA signals in NAc, compared with the corticolimbic nature of glutamate signals. That is, GABA signals would normally be delivered to spiny neurons in medial shell site by neighboring intrinsic NAc medium spiny neurons or interneurons, or by GABAergic afferent projections that arose from other subcortical structures such as ventral pallidum, extended amygdala (e.g. BNST), lateral hypothalamus or brainstem (Brog et al., 1993; Sun \& Cassell, 1993; Churchill \& Kalivas, 1994; Vanbockstaele \& Pickel, 1995; Meredith et al., 2008).

Environmental ambience signals by comparison might ordinarily be delivered to NAc largely by 'top-down' corticolimbic glutamatergic projections from prefrontal cortex, or other glutamatergic inputs from cortex-related structures such as basolateral amygdala, hippocampus or thalamus (Beckstead, 1979; Christie et al., 1987; Fuller et al., 1987; Goldin et al., 2008; Richard \& Berridge, 2013). Intrinsic subcortical GABA signals therefore might be somewhat 'bottom- up' and relatively autonomous from 'top-down' environmental modulation. By this view of GABAergic autonomy, the resistance of GABAergic release of appetitive and fearful motivation to changes in emotional environment reflects the resistance of emotional processes generated by intrinsically subcortical circuits to top-down modulation by factors that can influence corticolimbic regulation of emotion (Beauregard et al., 2001; Russell, 2003; Davidson, 2004; Barrett et al., 2007; Posner et al., 2007).

Likewise, while glutamate signals to NAc neurons interact closely with mesolimbic dopamine signals, often on the same spine (Cepeda et al., 1993; Calabresi et al., 1997; Brady \& O’Donnell, 2004; Tecuapetla et al., 2010), GABA signals to NAc neurons may be less modulated by co-occurring dopamine inputs. Although dopamine may modify pre-synaptic GABA release from NAc interneurons (Bracci et al., 2002; Centonze et al., 2002; Tecuapetla et al., 2007; Towers \& Hestrin, 2008), we are aware of no studies reporting that the post-synaptic hyperpolarizing effects of $\mathrm{GABA}_{\mathrm{A}}$ receptor activation on medium spiny neurons are modulated by dopamine. For example, dopamine transporter knock-down mice, which have increased levels of dopamine in the synapse, have normal GABAreceptor-mediated synaptic currents onto striatal medium spiny neurons (Wu et al., 2007). GABAergic generation of eating and fear by muscimol microinjections may mimic natural post-synaptic GABA signals that do not depend on post-synaptic dopamine, and so it is understandable that intense muscimol-generated motivations are not impaired by blockade of D1 or D2 inputs. The dopamine-independent nature of GABAergic eating and fear is also consistent with subcortical GABAergic generation of 'liking' or disgust reactions that reflect intense hedonic impact, psychological processes which are relatively independent of mesolimbic dopamine (Peciña et al., 


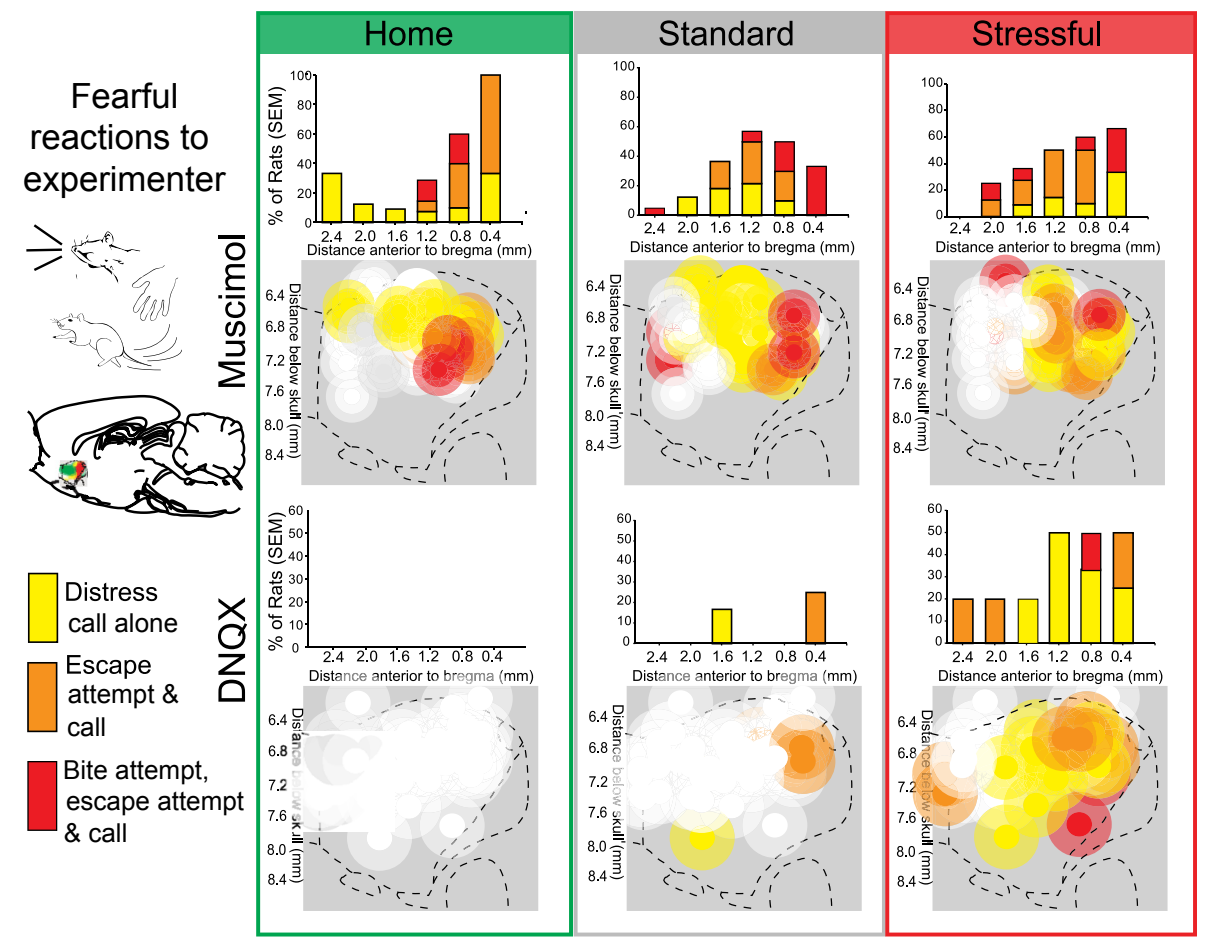

FIG. 6. Effect of changing environmental ambience on defensive reactions to the experimenter produced by DNQX or muscimol. Fos plume maps $(n=59)$ of the generation of defensive reactions to the experimenter by muscimol (top) or DNQX (bottom) in the Home (left), Standard (middle) or Stressful (right) environments. Changing environment between the familiar Home or the aversive Stressful environments had no effect on defensive reactions produced by muscimol, which were robustly generated regardless of environment ambience. In the Stressful environment, defensive reactions to the experimenter produced by DNQX treading were generated at more rostral locations in shell, but were nearly eliminated by testing in a Home environment. Histogram bars show the percentage of rats emitting distress calls (yellow), distress calls and escape attempts (red), and distress calls, escape attempts and bite attempts (red) at each rostrocaudal level as marked along the medial shell.

1997, 2003; Berridge \& Robinson, 1998; Faure et al., 2010). Here, muscimol microinjection would mimic post-synaptic GABA release effects by directly binding to $\mathrm{GABA}_{\mathrm{A}}$ receptors in medial shell, and hence may have acted downstream of dopamine modulation. This account of muscimol microinjection effects does not exclude the possibility that, under natural conditions, changes in dopamine may modulate motivated behaviors by presynaptic modulation of endogenous GABA release in NAc (Bracci et al., 2002; Centonze et al., 2002; Tecuapetla et al., 2007; Towers \& Hestrin, 2008).

It is also possible that hyperpolarizations produced by GABAergic muscimol are more robust or intense at the neuronal level than hyperpolarizations by DNQX glutamatergic blockade (Koos et al., 2004), which merely diminishes 'up states' to suppress excitatory postsynaptic potentials (Meredith et al., 1993; Pennartz et al., 1994; Kiyatkin \& Rebec, 1999; Meredith, 1999; O’Donnell, 1999; Suwabe et al., 2008; Jeun et al., 2009). GABAergic signaling may more potently inhibit NAc medium spiny neurons by acting directly to open $\mathrm{Cl}^{-}$channels on soma or proximal dendrites (Sun \& Cassell, 1993; Johnson et al., 1994; Behrends et al., 2002; Goetz et al., 2007), whereas glutamatergic AMPA receptors are localized more distally at the ends of dendrite spines (Meredith et al., 1990; Sesack \& Pickel, 1992; Johnson et al., 1994; Chen et al., 1998), allowing dopamine released on spines or dendrites to play a greater role.

\section{NAc keyboard pattern}

Just as a musical keyboard generates many different notes, depending on which key is tapped, the NAc 'affective keyboard' for generating desire vs. dread released many different ratios of appetitive to fearful motivated behaviors depending on the precise rostrocaudal position of a GABAergic or glutamatergic microinjection. Our data suggest that the anterior-posterior length of medial shell, which extends nearly $2.5 \mathrm{~mm}$, contains multiple 'key' levels or a ratio continuum of substrates for opposite motivations. That is, each microinjection here released its own individualized ratio of valencegenerating substrates (i.e. either at least three detectable rostrocaudal levels or a gradual continuum capable of producing many more potential ratios).

The different ratios appear to be generated by mechanisms contained close to microinjection sites, rather than due to distant drug diffusion to another part of medial shell. That is suggested especially by the relatively small diameter of muscimol Fos plumes, which were just under $0.5 \mathrm{~mm}(0.24 \mathrm{~mm}$ radius $)$, as well as the just slightly larger diameter of DNQX Fos plumes, just under $0.8 \mathrm{~mm}$ (0.38 mm radius). As a caveat, Fos plumes are not infallible indicators for neurobiological impact spread. However, plumes do at least provide some objective information on how far a microinjected drug's impact may extend. It therefore seems worthwhile to consider the implications of measured plume spread, at least given the absence of evidence that muscimol microinjection impact extends any further. One implication of relatively small $0.5-0.8 \mathrm{~mm}$ diameters of impact spread is not only that substrates for both motivations must have been reachable within that spread from most shell positions, but also that the different ratio mechanisms must have been locally contained.

Why can a simpler mechanism not fully account for our effects, say, in which the front half of the shell simply produced eating and the caudal half produced fearful behavior? The reason is that two 


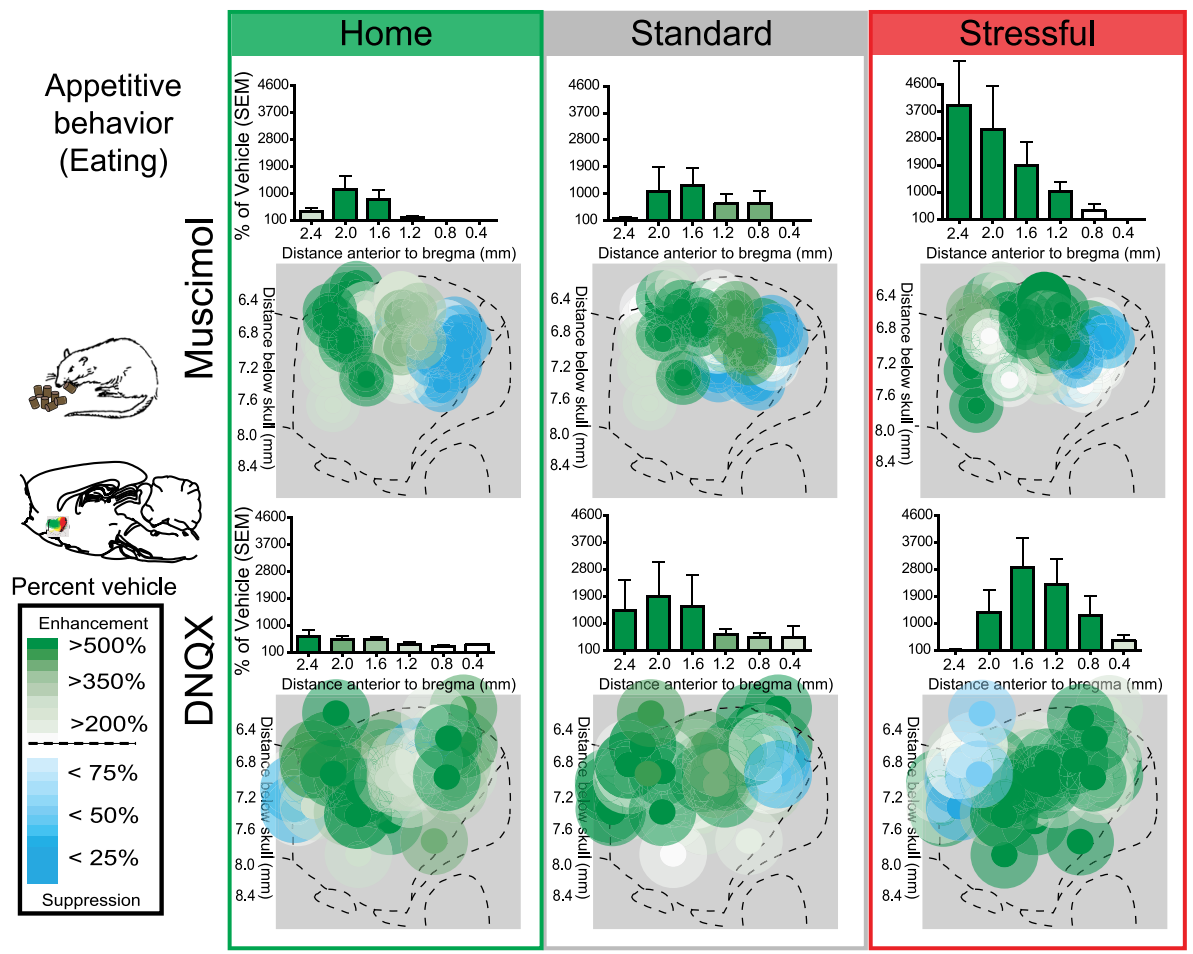

FIG. 7. Effect of changing environmental ambience on eating produced by DNQX or muscimol. Fos plume maps $(n=59)$ of the generation of eating by muscimol (top) or DNQX (bottom) in the Home (left), Standard (middle) or Stressful (right) environments. Changing environmental ambience had inconsistent effects on eating produced by DNQX or muscimol. Histogram bars show treading as a percentage of vehicle at each rostrocaudal level as marked along the medial shell (error bars $=$ SEM).
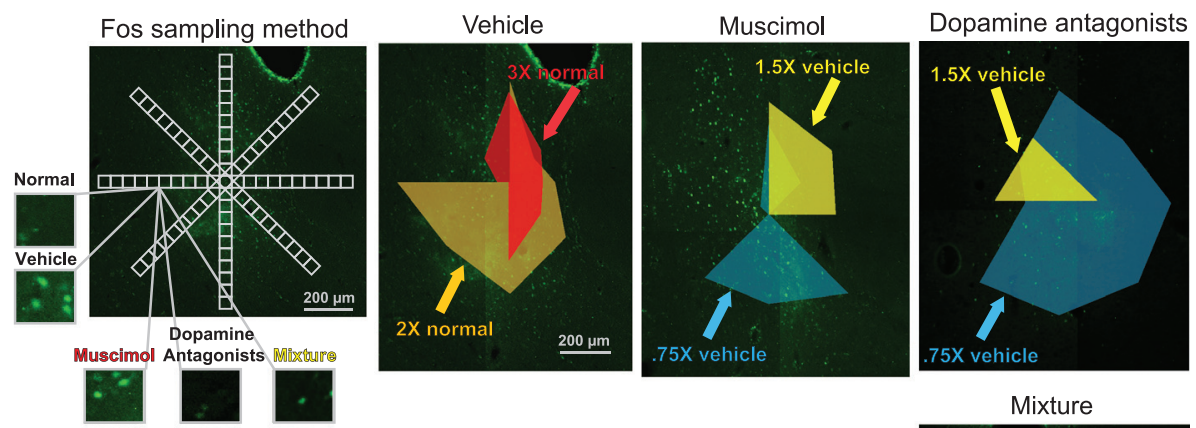

Mixture
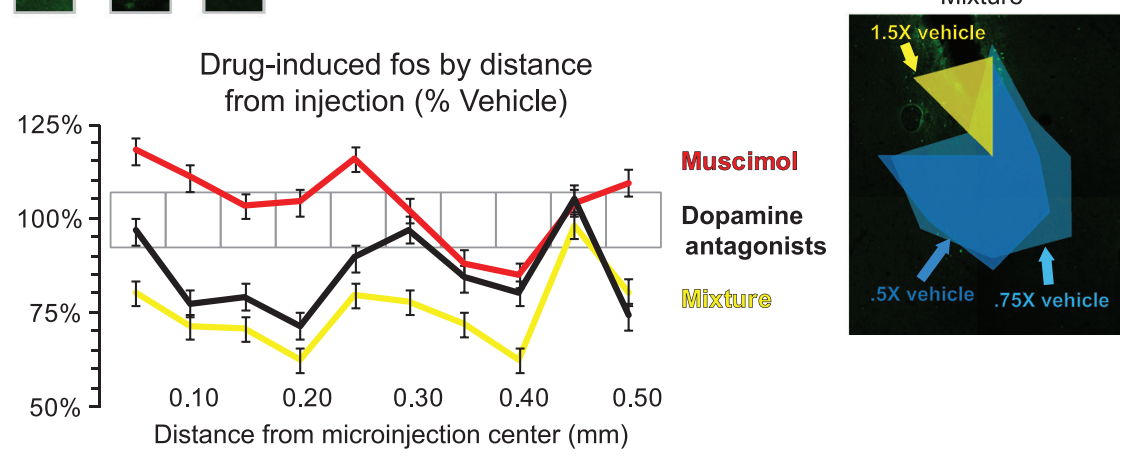

FIG. 8. Fos plume analysis. Fos expression was assessed following microinjections of vehicle, muscimol, dopamine antagonists alone, or a mixture of musci$\mathrm{mol}$ and dopamine antagonists. Fos-labeled cells were individually counted within successive blocks $(50 \times 50 \mu \mathrm{m})$, along eight radial arms emanating from the center of the site, with $10 \times$ magnification. For vehicle microinjections colors indicate levels of Fos expression of $3 \times($ red), and $2 \times($ orange) levels of Fos expression found in normal (uninjected) tissue. For drug microinjections, colors indicate levels of Fos expression of $1.5 \times(y e l l o w), 0.75 \times($ light blue) and $0.50 \times$ (darker blue) vehicle level Fos expression. Line graphs show that muscimol (red) reduced Fos expression approximately $\sim 0.35$ mm away from the microinjection center, and that dopamine antagonists (black) and mixture (yellow) reduced Fos expression from 0.15 mm to 0.40 mm away from the microinjection center 
discrete non-overlapping substrates cannot easily account for the multiple quantitative ratios of appetitive and defensive behavior seen as sites moved rostral to caudal, or for the capacity for most sites in medial shell to release both appetitive and fearful motivations. That is, the two halves of medial shell did not respond as simple qualitative on/off mechanisms. Instead, moves of position along the AP axis produced proportional shifts in the ratio of intense motivated behaviors.

Perhaps the most compelling evidence against a simple two-key mechanism for differently valenced motivations contained in the two halves of NAc shell are the glutamatergic data which confirmed that each half of NAc medial shell must also contain at least some substrates for generating its opposite-valenced motivation. That is, the rostral half of medial shell must also contain at least sparse fearreleasing substrates, and the caudal shell must also contain at least sparse eating-releasing substrates. This is demonstrated by the ability of glutamate-disrupting DNQX microinjections fully contained in the rostral half of shell to release fear (in a stressful environment), and for microinjections contained in the caudal half to release eating (in a comfortable home environment). A qualitative two-mechanism hypothesis cannot easily explain such reversal patterns.

One way of reconciling all these observations would be to conceive of NAc eating/fear mechanisms as two cloud continuums for desire/dread substrates extending through medial shell. One substrate is densest near the rostral pole for generating appetitive eating, but stretches in declining density nearly to the caudal pole. A second is densest near the caudal pole for generating fearful responses, but stretches in declining density nearly to the rostral pole. The two mechanisms would overlap in varying densities or efficacies throughout nearly the entire $2.5-\mathrm{mm}$ rostrocaudal extent of medial shell. As consequence, the 'affective keyboard' pattern of behavioral ratios we observed here would be produced by the relative densities of substrates within the diameter of each single microinjection along the AP continuum. Of course, the challenge of identifying what this actually means in terms of neuronal mechanisms for determining the valence of intense motivations released by a microinjection remains a major task for the future.

\section{Disinhibition of rostral vs. caudal NAc shell outputs}

The neuronal mechanism by which NAc inhibitions generated motivations is likely to be release or disinhibition of motivation generators in target structures, as NAc projection neurons are primarily GABAergic, and would be expected to tonically inhibit downstream neurons (Taber \& Fibiger, 1997; Kelley, 1999; Roitman et al., 2008; Krause et al., 2010). Temporary inhibition of NAc neurons by GABAergic muscimol microinjection would remove downstream GABAergic inhibition, releasing a subpopulation of recipient neurons in a target structure to become relatively excited and generate intense motivations. Downstream target structures for potential release include ventral pallidum, ventral tegmentum and lateral hypothalamus, which all receive GABAergic inputs that originate throughout the rostrocaudal extent of medial shell (Mogenson et al., 1983; Zahm \& Heimer, 1990; Heimer et al., 1991). Caudal NAc shell also probably projects to the extended amygdala, including the central and medial amygdala, the ventrolateral bed nucleus of the stria terminalis and the sublenticular gray, as well as potentially to the retrorubral area, the substantia nigra and the parabrachial nucleus (Heimer et al., 1991; Zahm \& Heimer, 1993; Usuda et al., 1998). Rostral shell projects to specific targets including particular subregions of the lateral preoptic area, lateral hypothalamus and ventral pallidum (Usuda et al., 1998; Thompson \& Swanson, 2010; Zahm et al., 2013). Similarly, relative inhibitions of localized NAc neurons by DNQX microinjections, which block excitatory glutamate inputs to NAc such as from corticolimbic projections, might also disinhibit distinct subpopulations in target structures to release the generation of intense motivations. Rostrocaudal differences in potential targets of release may therefore contribute to different ratios observed along the NAc rostrocaudal axis in generation of fear vs. eating.

\section{Conclusions}

The NAc generation of intense motivations by localized GABA inhibition obeys different rules from generation by glutamatergic disruption regarding dopamine and environmental retuning, even though GABA inhibition and glutamate disruption both produce similar appetitive and fearful behaviors organized along the same NAc rostrocaudal gradient. The GABAergic generation of intense eating and fearful motivations is solely determined by the anatomical position of the muscimol microinjections in NAc medial shell. The valence and intensity of GABAergic appetitive and fearful motivations are relatively immune to modulation by environmental ambience and free of any need for local dopamine inputs. By contrast, the keyboard pattern of desire and dread generated by glutamate disruption is easily retuned by changes in emotional environment, as well as highly dependent on endogenous dopamine inputs.

This distinction between glutamatergic motivations that are modifiable via top-down psychological and mesolimbic dopamine control, compared with robust GABAergic motivational states that are not, may have implications for understanding mechanisms of some psychiatric disorders and psychopathologies of emotional well-being. Flips in the valence of pathologically intense motivational salience may occur more easily when they primarily involve NAc corticolimbic or other glutamatergic mechanisms, than when motivations are generated by subcortical GABAergic mechanisms that are intrinsic to NAc. Glutamate/dopamine modifiability may relate to why the incentive salience of amphetamine addiction can flip valence from appetitive to the fearful salience of amphetamine psychosis, or why schizophrenic patients can have not only heightened fearful salience of paranoia but may also show exaggerated incentive salience for appetitive stimuli (Elman et al., 2006; Featherstone et al., 2007; Jensen et al., 2008; Howes \& Kapur, 2009). In contrast, motivations released by GABAergic inhibitions in NAc-related circuits may be more anatomically coded, consistently valenced, hedonically laden and less amenable to regulation by top-down control (Faure et al., 2010; Watson \& Naragon-Gainey, 2010).

\section{Acknowledgements}

This research was supported by National Institutes of Health Grants (DA015188 and MH63649 to K.C.B.) and by a National Research Service Award fellowship to J.M.R. (MH090602). We thank Aaron Garcia for assistance with immunohistochemistry.

\section{Abbreviations}

ACSF, artificial cerebrospinal fluid; AMPA, $\alpha$-amino-3-hydroxy-5-methylisoxazole-4-propionic acid; DNQX, 6,7-dinitroquinoxaline-2,3-dione; GABA, $\gamma$-aminobutyric acid; NAc, nucleus accumbens.

\section{References}

Aldridge, J.W., Berridge, K.C., Herman, M. \& Zimmer, L. (1993) Neuronal coding of serial order: syntax of grooming in the neostriatum. Psychol. Sci., 4, 391-395. 
Barrett, L.F., Mesquita, B., Ochsner, K.N. \& Gross, J.J. (2007) The experience of emotion. Апnи. Rev. Psychol., 58, 373-403.

Beauregard, M., Levesque, J. \& Bourgouin, P. (2001) Neural correlates of conscious self-regulation of emotion. J. Neurosci., 21, U11-U16.

Beckstead, R.M. (1979) Autoradiographic examination of cortico-cortical and sub-cortical projections of the mediodorsal-projection (prefrontal) cortex in the rat. J. Comp. Neurol., 184, 43-62.

Behrends, J.C., Lambert, J.D.C. \& Jensen, K. (2002) Repetitive activation of postsynaptic GABA(A) receptors by rapid, focal agonist application onto intact rat striatal neurones in vitro. Pflugers Arch., 443, 707-712.

Berridge, K.C. \& Robinson, T.E. (1998) What is the role of dopamine in reward: hedonic impact, reward learning, or incentive salience? Brain Res. Rev., 28, 309-369.

Bracci, E., Centonze, D., Bernardi, G. \& Calabresi, P. (2002) Dopamine excites fast-spiking interneurons in the striatum. J. Neurophysiol., 87, 2190-2194.

Brady, A.M. \& O'Donnell, P. (2004) Dopaminergic modulation of prefrontal cortical input to nucleus accumbens neurons in vivo. J. Neurosci., 24 1040-1049

Brog, J.S., Salyapongse, A., Deutch, A.Y. \& Zahm, D.S. (1993) The patterns of afferent innervation of the core and shell in the 'accumbens' part of the rat ventral striatum: immunohistochemical detection of retrogradely transported fluoro-gold. J. Comp. Neurol., 338, 255-278.

Calabresi, P., Pisani, A., Centonze, D. \& Bernardi, G. (1997) Synaptic plasticity and physiological interactions between dopamine and glutamate in the striatum. Neurosci. Biobehav. R., 21, 519-523.

Centonze, D., Picconi, B., Baunez, C., Borrelli, E., Pisani, A., Bernardi, G. \& Calabresi, P. (2002) Cocaine and amphetamine depress striatal GABAergic synaptic transmission through D2 dopamine receptors. Neuropsychopharmacol., 26, 164-175.

Cepeda, C., Buchwald, N.A. \& Levine, M.S. (1993) Neuromodulatory actions of dopamine in the neostriatum are dependent upon the excitatory amino acid receptor subtypes activated. Proc. Natl. Acad. Sci. USA, 90, 9576-9580.

Chen, Q., Veenman, L., Knopp, K., Yan, Z., Medina, L., Song, W.J., Surmeier, D.J. \& Reiner, A. (1998) Evidence for the preferential localization of glutamate receptor-1 subunits of AMPA receptors to the dendritic spines of medium spiny neurons in rat striatum. Neuroscience, 83, 749761.

Christie, M.J., Summers, R.J., Stephenson, J.A., Cook, C.J. \& Beart, P.M. (1987) Excitatory amino-acid projections to the nucleus accumbens-septi in the rat: a retrograde transport study utilizing $\mathrm{D}[\mathrm{H}-3]$ aspartate and $[\mathrm{H}-3]$ GABA. Neuroscience, 22, 425-439.

Churchill, L. \& Kalivas, P.W. (1994) A topographically organized gammaaminobutyric acid projection from the ventral pallidum to the nucleus accumbens in the rat. J. Comp. Neurol., 345, 579-595.

Coss, R.G. \& Owings, D.H. (1978) Snake-directed behavior by snake naive and experienced California ground squirrels in a simulated burrow. Z. Tierpsychol., 48, 421-435.

Davidson, R.J. (2004) Well-being and affective style: neural substrates and biobehavioural correlates. Philos. T. Roy. Soc. B, 359, 1395-1411.

Dragunow, M. \& Faull, R. (1989) The use of c-fos as a metabolic marker in neuronal pathway tracing. J. Neurosci. Meth., 29, 261-265.

Elman, I., Borsook, D. \& Lukas, S.E. (2006) Food intake and reward mechanisms in patients with schizophrenia: implications for metabolic disturbances and treatment with second-generation antipsychotic agents. Neuropsychopharmacol., 31, 2091-2120.

Faure, A., Reynolds, S.M., Richard, J.M. \& Berridge, K.C. (2008) Mesolimbic dopamine in desire and dread: enabling motivation to be generated by localized glutamate disruptions in nucleus accumbens. J. Neurosci., 28, 7184-7192

Faure, A., Richard, J.M. \& Berridge, K.C. (2010) Desire and dread from the nucleus accumbens: cortical glutamate and subcortical GABA differentially generate motivation and hedonic impact in the rat. PLOS ONE, 5, e11223.

Featherstone, R.E., Kapur, S. \& Fletcher, P.J. (2007) The amphetamineinduced sensitized state as a model of schizophrenia. Prog. NeuroPsychoph., 31, 1556-1571.

Fuller, T.A., Russchen, F.T. \& Price, J.L. (1987) Sources of presumptive glutamatergic aspartergic afferents to the rat ventral striatopallidal region. J. Comp. Neurol., 258, 317-338.

Goetz, T., Arslan, A., Wisden, W. \& Wulff, P. (2007) GABA(A) receptors: structure and function in the basal ganglia. Prog. Brain Res., 160, $21-41$.

Goldin, P.R., McRae, K., Ramel, W. \& Gross, J.J. (2008) The neural bases of emotion regulation: reappraisal and suppression of negative emotion. Biol. Psychiat., 63, 577-586.
Heimer, L., Zahm, D.S., Churchill, L., Kalivas, P.W. \& Wohltmann, C. (1991) Specificity in the projection patterns of accumbal core and shell in the rat. Neuroscience, 41, 89-125.

Howes, O.D. \& Kapur, S. (2009) The dopamine hypothesis of schizophrenia: version III-the final common pathway. Schizophrenia Bull., 35 , $549-562$.

Jensen, J., Willeit, M., Zipursky, R.B., Savina, I., Smith, A.J., Menon, M., Crawley, A.P. \& Kapur, S. (2008) The formation of abnormal associations in schizophrenia: neural and behavioral evidence. Neuropsychopharmacol., 33, 473-479.

Jeun, S.H., Cho, H.S., Kim, K.J., Li, Q.Z. \& Sung, K.W. (2009) Electrophysiological characterization of AMPA and NMDA receptors in rat dorsal striatum. Korean J. Physiol. Pha., 13, 209-214.

Johnson, L.R., Aylward, R.L.M. \& Totterdell, S. (1994) Synaptic organization of the amygdalar input to the nucleus-accumbens in the rat. In Percheron, G., McKenzie, J.S. \& Feger, J. (Eds) Basal Ganglia IV - New Ideas and Data on Structure and Function. Plenum Press, New York, pp. 109-114.

Kelley, A.E. (1999) Functional specificity of ventral striatal compartments in appetitive behaviors. Ann. NY Acad. Sci., 877, 71-90.

Kelley, A.E., Bakshi, V.P., Fleming, S. \& Holahan, M.R. (2000) A pharmacological analysis of the substrates underlying conditioned feeding induced by repeated opioid stimulation of the nucleus accumbens. Neuropsychopharmacol., 23, 455-467.

Kiyatkin, E.A. \& Rebec, G.V. (1999) Modulation of striatal neuronal activity by glutamate and GABA: iontophoresis in awake, unrestrained rats. Brain Res., 822, 88-106.

Koos, T., Tepper, J.M. \& Wilson, C.J. (2004) Comparison of IPSCs evoked by spiny and fast-spiking neurons in the neostriatum. J. Neurosci., 24 7916-7922.

Krause, M., German, P.W., Taha, S.A. \& Fields, H.L. (2010) A pause in nucleus accumbens neuron firing is required to initiate and maintain feeding. J. Neurosci., 30, 4746-4756.

Maldonado-Irizarry, C.S., Swanson, C.J. \& Kelley, A.E. (1995) Glutamate receptors in the nucleus accumbens shell control feeding behavior via the lateral hypothalamus. J. Neurosci., 15, 6779-6788.

Meredith, G.E. (1999) The synaptic framework for chemical signaling in nucleus accumbens. Ann. NY Acad. Sci., 877, 140-156.

Meredith, G.E., Wouterlood, F.G. \& Pattiselanno, A. (1990) Hippocampal fibers make synaptic contacts with glutamate decarboxylase-immunoreactive neurons in the rat nucleus accumbens. Brain Res., 513, 329-334.

Meredith, G.E., Pennartz, C.M. \& Groenewegen, H.J. (1993) The cellular framework for chemical signalling in the nucleus accumbens. Prog. Brain Res., 99, 3-24.

Meredith, G.E., Baldo, B.A., Andrezjewski, M.E. \& Kelley, A.E. (2008) The structural basis for mapping behavior onto the ventral striatum and its subdivisions. Brain Struct. Funct., 213, 17-27.

Mogenson, G.J., Swanson, L.W. \& Wu, M. (1983) Neural projections from nucleus accumbens to globus pallidus, substantia innominata, and lateral preoptic-lateral hypothalamic area: an anatomical and electrophysiological investigation in the rat. J. Neurosci., 3, 189-202.

O'Donnell, P. (1999) Ensemble coding in the nucleus accumbens. Psychobiology, 27, 187-197.

Paxinos, G. \& Watson, C. (2007) The rat brain in stereotaxic coordinates. Academic Press, New York.

Peciña, S., Berridge, K.C. \& Parker, L.A. (1997) Pimozide does not shift palatability: separation of anhedonia from sensorimotor suppression by taste reactivity. Pharmacol. Biochem. Be., 58, 801-811.

Peciña, S., Cagniard, B., Berridge, K.C., Aldridge, J.W. \& Zhuang, X. (2003) Hyperdopaminergic mutant mice have higher 'wanting' but not 'liking' for sweet rewards. J. Neurosci., 23, 9395-9402.

Pennartz, C.M., Groenewegen, H.J. \& Lopes da Silva, F.H. (1994) The nucleus accumbens as a complex of functionally distinct neuronal ensembles: an integration of behavioural, electrophysiological and anatomical data. Prog. Neurobiol., 42, 719-761.

Posner, M.I., Rothbart, M.K., Sheese, B.E. \& Tang, Y. (2007) The anterior cingulate gyrus and the mechanism of self-regulation. Cogn. Affect. Behav. Ne., 7, 391-395.

Reynolds, S.M. \& Berridge, K.C. (2001) Fear and feeding in the nucleus accumbens shell: rostrocaudal segregation of GABA-elicited defensive behavior versus eating behavior. J. Neurosci., 21, 3261-3270.

Reynolds, S.M. \& Berridge, K.C. (2002) Positive and negative motivation in nucleus accumbens shell: bivalent rostrocaudal gradients for GABA-elicited eating, taste 'liking'/'disliking' reactions, place preference/avoidance, and fear. J. Neurosci., 22, 7308-7320. 
Reynolds, S.M. \& Berridge, K.C. (2003) Glutamate motivational ensembles in nucleus accumbens: rostrocaudal shell gradients of fear and feeding. Eur. J. Neurosci., 17, 2187-2200.

Reynolds, S.M. \& Berridge, K.C. (2008) Emotional environments retune the valence of appetitive versus fearful functions in nucleus accumbens. Nat Neurosci., 11, 423-425.

Richard, J.M. \& Berridge, K.C. (2011) Nucleus accumbens dopamine/glutamate interaction switches modes to generate desire versus dread: $D(1)$ alone for appetitive eating but $\mathrm{D}(1)$ and $\mathrm{D}(2)$ together for fear. J. Neurosci., 31, 12866-12879.

Richard, J.M. \& Berridge, K.C. (2013) Prefrontal cortex modulates desire and dread generated by nucleus accumbens glutamate disruption. Biol. Psychiat., 73, 360-370.

Roitman, M.F., Wheeler, R.A., Wightman, R.M. \& Carelli, R.M. (2008) Real-time chemical responses in the nucleus accumbens differentiate rewarding and aversive stimuli. Nat. Neurosci., 11, 1376-1377.

Russell, J.A. (2003) Core affect and the psychological construction of emotion. Psychol. Rev., 110, 145-172.

Sesack, S.R. \& Pickel, V.M. (1992) Prefrontal cortical efferents in the rat synapse on unlabeled neuronal targets of catecholamine terminals in the nucleus accumbens septi and on dopamine neurons in the ventral tegmental area. J. Comp. Neurol., 320, 145-160.

Stratford, T.R. \& Kelley, A.E. (1997) GABA in the nucleus accumbens shell participates in the central regulation of feeding behavior. J. Neurosci., 17 4434-4440.

Sun, N. \& Cassell, M.D. (1993) Intrinsic GABAergic neurons in the rat central extended amygdala. J. Comp. Neurol., 330, 381-404.

Suwabe, T., Fukami, H. \& Bradley, R.M. (2008) Synaptic responses of neurons controlling the parotid and von Ebner salivary glands in rats to stimulation of the solitary nucleus and tract. J. Neurophysiol., 99, 1267-1273.

Taber, M.T. \& Fibiger, H.C. (1997) Feeding-evoked dopamine release in the nucleus, accumbens: regulation by glutamatergic mechanisms. Neuroscience, 76, 1105-1112.

Tecuapetla, F., Carrillo-Reid, L., Bargas, J. \& Galarraga, E. (2007) Dopaminergic modulation of short-term synaptic plasticity at striatal inhibitory synapses. Proc. Natl. Acad. Sci. USA, 104, 10258-10263.
Tecuapetla, F., Patel, J.C., Xenias, H., English, D., Tadros, I., Shah, F., Berlin, J., Deisseroth, K., Rice, M.E., Tepper, J.M. \& Koos, T. (2010) Glutamatergic signaling by mesolimbic dopamine neurons in the nucleus accumbens. J. Neurosci., 30, 7105-7110.

Thompson, R.H. \& Swanson, L.W. (2010) Hypothesis-driven structural connectivity analysis supports network over hierarchical model of brain architecture. Proc. Natl. Acad. Sci. USA, 107, 15235-15239.

Towers, S.K. \& Hestrin, S. (2008) D1-like dopamine receptor activation modulates GABAergic inhibition but not electrical coupling between neocortical fast-spiking interneurons. J. Neurosci., 28, 2633-2641.

Treit, D., Pinel, J.P. \& Fibiger, H.C. (1981) Conditioned defensive burying: a new paradigm for the study of anxiolytic agents. Pharmacol. Biochem. Be., 15, 619-626.

Usuda, I., Tanaka, K. \& Chiba, T. (1998) Efferent projections of the nucleus accumbens in the rat with special reference to subdivision of the nucleus: biotinylated dextran amine study. Brain Res., 797, 73-93.

Vanbockstaele, E.J. \& Pickel, V.M. (1995) GABA-containing neurons in the ventral tegmental area project to the nucleus-accumbens in rat brain. Brain Res., 682, 215-221.

Watson, D. \& Naragon-Gainey, K. (2010) On the specificity of positive emotional dysfunction in psychopathology: evidence from the mood and anxiety disorders and schizophrenia/schizotypy. Clin. Psychol. Rev., 30, 839-848.

Wu, N., Cepeda, C., Zhuang, X. \& Levine, M.S. (2007) Altered corticostriatal neurotransmission and modulation in dopamine transporter knock-down mice. J. Neurophysiol., 98, 423-432.

Zahm, D.S. \& Heimer, L. (1990) Two transpallidal pathways originating in the rat nucleus accumbens. J. Comp. Neurol., 302, 437-446.

Zahm, D.S. \& Heimer, L. (1993) Specificity in the efferent projections of the nucleus accumbens in the rat: comparison of the rostral pole projection patterns with those of the core and shell. J. Comp. Neurol., 327, 220 -232 .

Zahm, D.S., Parsley, K.P., Schwartz, Z.M. \& Cheng, A.Y. (2013) On lateral septum-like characteristics of outputs from the accumbal hedonic 'hotspot' of Peciña and Berridge with commentary on the trasitional nature of basal forebrain 'boundaries'. J. Comp. Neurol., 521, 50-68. 